\title{
Muséologies
}

Les cahiers d'études supérieures

\section{« Le Musée national de Beyrouth et Beit Beyrouth : ou de la provocation de la mémoire »}

\section{Claudia Polledri}

Volume 8, numéro 2, 2016

Les nouveaux paradigmes

URI : https://id.erudit.org/iderudit/1050760ar

DOI : https://doi.org/10.7202/1050760ar

Aller au sommaire du numéro

Éditeur(s)

Association Québécoise de Promotion des Recherches Étudiantes en

Muséologie (AQPREM)

ISSN

1718-5181 (imprimé)

1929-7815 (numérique)

Découvrir la revue

Citer ce compte rendu

Polledri, C. (2016). Compte rendu de [« Le Musée national de Beyrouth et Beit Beyrouth : ou de la provocation de la mémoire »]. Muséologies, 8(2), 63-77. https://doi.org/10.7202/1050760ar

Tous droits réservés (C Association Québécoise de Promotion des Recherches Étudiantes en Muséologie (AQPREM), 2018
Ce document est protégé par la loi sur le droit d'auteur. L'utilisation des services d'Érudit (y compris la reproduction) est assujettie à sa politique d'utilisation que vous pouvez consulter en ligne.

https://apropos.erudit.org/fr/usagers/politique-dutilisation/ 
Carnet deux

\section{"Le Musée national de Beyrouth et Beit Beyrouth: ou de la provocation de la mémoire"}

Claudia Polledri

« J'ai 4000 ans, j'ai 5000 ans.

Je suis la ville, je suis Beyrouth.

Une éponge dans la main, et dans l'autre une craie;

d'une main, j'écris des histoires d'enfant, je construis des avenues, des palais

et de l'autre main, j'efface les enfants, les avenues, et les palais. »

Une vie suspendue, Jocelyne Saab 
Postdoctorante et chargée de cours au Département d'histoire de l'art et d'études cinématographiques de l'Université de Montréal, Claudia Polledri est titulaire d'un doctorat en littérature comparée de l'Université de Montréal portant sur les représentations photographiques de Beyrouth (1982-2011) et sur le rapport entre photographie et histoire. Elle a été chercheuse invitée au LLA-CRÉATIS (2016) et depuis 2015 assure la coordination scientifique du CR Ialt. Sa recherche actuelle porte sur la «Fondation arabe pour l'image" et sur la démarche de patrimonialisation de la photographie au Maghreb et au Moyen-Orient. 
Fortement marqué d'abord par la guerre civile (1975-1990) et ensuite par le conflit avec Israël (2006), le tissu urbain beyrouthin a continué, pendant ces dernières années, de se transformer à coup de destructions et reconstructions, faisant de la capitale libanaise un être changeant, le profil en constante mutation. Cette métamorphose, toutefois, est loin de faire consensus. Parmi ces interventions, le point le plus controversé concerne sans doute la reconstruction du centre-ville ${ }^{1}$. Débuté dans les années 1990 à l'initiative de SOLIDERE², la société de reconstruction gérée par l'ex-premier ministre Rafiq Hariri, ce projet a donné lieu à un centre-ville vitrine, une sorte de copie de l'original, désormais déserté par les Libanais. Outre d'avoir effacé toute trace de l'histoire récente, cet énorme chantier a aussi provoqué des « dommages collatéraux » importants à un patrimoine archéologique ${ }^{3}$ déjà détérioré par les conflits. Mais les " pertes " subies par la ville - perte du noyau historique et de l'espace public qu'il représentait, perte des traces du conflit - ne s'arrêtent pas ici. Elles se poursuivent aujourd'hui à travers la démolition progressive du patrimoine architectural traditionnel libanais, au profit des intérêts des compagnies immobilières. Des quartiers «à caractère traditionnel ${ }^{4}$ " sont en effet en train de disparaître pour faire place à des immeubles modernes, pour la plupart résidentiels, souvent inhabités ${ }^{5}$.

Également concernés par ces mutations urbaines, les musées de la capitale libanaise tentent, au contraire, de résister à cette œuvre frénétique d'effacement des architectures et des mémoires. Ils sont en effet l'expression d'un travail de conservation de l'héritage culturel libanais ce qui, devant la spécificité de ce contexte, mérite d'être souligné. En plus des travaux de rénovation des musées déjà existants comme le Musée national de Beyrouth et, plus récemment, le Musée Sursok ${ }^{6}$, il faut signaler aussi l'établissement d'un nouveau musée. Il s'agit du cas de l'immeuble Barakat, un exemplaire unique d'architecture traditionnelle déjà condamné à la démolition, mais dont une forte mobilisation collective a réussi à obtenir la conservation et sa transformation en Beit Beirut ${ }^{7}$ (La maison de Beyrouth), le nouveau musée dédié à l'his-

1 Parmi les critiques les plus significatives, on signale celle de Adonis. Beirut la non città, San Giorgio a Cremano, Éditions Medusa, 2007.

2 SOLIDERE est l'acronyme de SOciété LIbanaise DE REconstruction. À ce propos, voir : Liliane Buccianti-Barakat, "Le centre-ville de Beyrouth ou un patrimoine réinventé? ", Habiter le patrimoine, (GRAVARI-BARBAS Maria dir.), Rennes: Presses universitaires de Rennes, 2005, p. 125-144. 3 Voir les textes de: AL-KASSIM Dina, "Crise de l'invisible. Découvrir l'esthétique de l'hystérie dans la politique de l'art et l'archéologie de la nouvelle Beyrouth ", Parachute, $\mathrm{n}^{\circ}$ 108, 2002, p. 147-163; RASCHKA Marylin, " Beirut Digs out ", Archaeological Ethics, (VITELLI Karen D., COLWELLCHANTHAPHONH Chip dir.), Toronto, Altamira Press, 2007, p. 96-102. À ce propos, il faut aussi signaler le nouveau projet muséal Beirut City Museum qui devrait accueillir les collections archéologiques issues des fouilles du centre-ville. Ce projet, financé par SOLIDERE et un fonds koweïtien, et voulu par le ministère de la Culture, devrait avoir lieu à Beyrouth dans deux ans et serait signé par l'architecte Renzo Piano: http://www.lorientlejour.com/article/1000223/un-museegriffe-renzo-piano-dans-deux-ans-a-beyrouth.html.

4 C'est l'expression qu'on trouve sur les panneaux affichés dans certains quartiers de Beyrouth: Gemmayzeh, Achrafieh, Zokak El Blat et bien d'autres..

5 À ce propos, on signale le film documentaire Bonjour Beyrouth réalisé par le journaliste Georges Salibi qui expose l'histoire d'un patrimoine qui se transforme en propriétés à vendre et aussi l'organisation non gouvernementale " Save Beirut Heritage " qui veille à la préservation du patrimoine architectural de la ville: http://savebeirutheritage.org/about. 6 Au sujet de la réouverture du musée Sursok, voir : http:/| www.lorientlejour.com/article/947662/la-bienheureuse-resurrection-du-musee-sursock.html.

7 Pour obtenir plus d'information, voir le site dédié au projet: http://www.beitbeirut.org. 


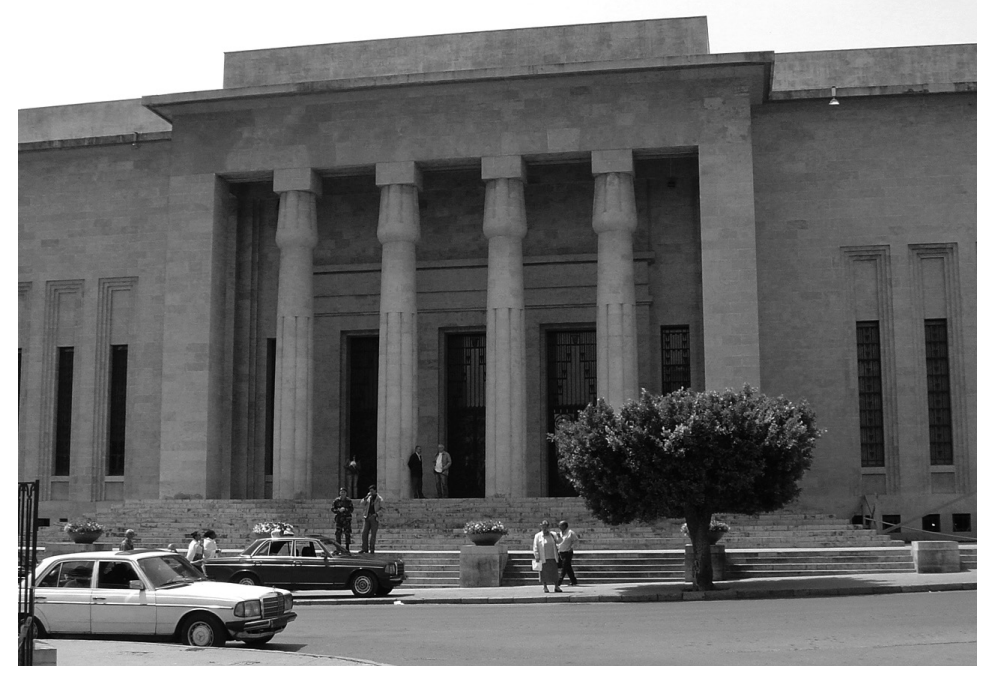

Le Musée national de Beyrouth

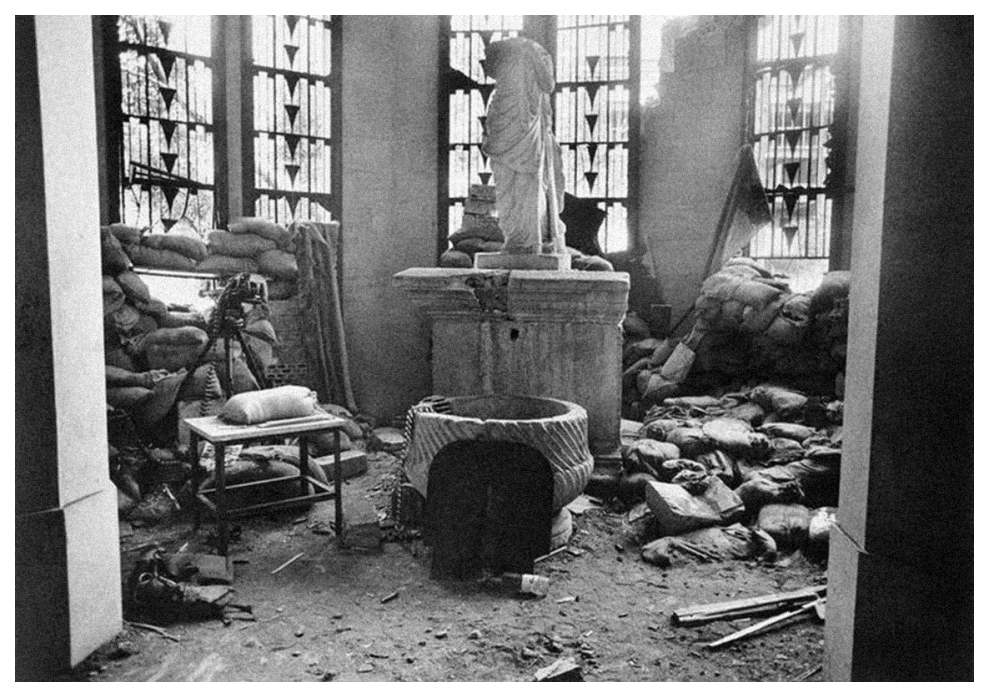

L'état du Musée national après le conflit (1990) 
toire de la ville. Un choix où l'enjeu de la conservation du patrimoine rencontre une forme de nécessité à sauvegarder la mémoire du conflit civil ; façon de résister à l'amnésie urbaine et collective engendrée par l'amnistie de $1989^{\circ}$, façon aussi de confier à l'institution muséale la conservation d'un patrimoine en voie de disparition.

À travers les exemples du Musée national et de Beit Beyrouth, il s'agit de montrer la " provocation de la mémoire 9 " à laquelle ils donnent lieu en ouvrant une troisième voie entre celle de la destruction et celle de la reconstruction, une voie difficile à se mettre en place dans le contexte libanais, notamment sur le plan institutionnel, celle de la transmission de la mémoire.

\section{Le Musée national de Beyrouth}

Conçu à l'époque du mandat français, le Musée national de Beyrouth ouvre ses portes le 27 mai 1942, en présence du président de la République du Liban Alfred Naccache ${ }^{10}$. Réunissant, dès son ouverture, environ 1300 objets de la préhistoire à l'époque mamelouke, la collection continue de grandir notamment entre les années 1960 et 1970, la période la plus prolifique du point de vue des fouilles archéologiques menées par la Direction générale des antiquités. Malheureusement, l'éclatement du conflit en 1975 a conduit à l'interruption des travaux mais, surtout, il a rendu plus difficile la protection des collections.

En raison de son emplacement sur la route de Damas, ligne de démarcation entre Beyrouth-Est et Beyrouth-Ouest pendant le conflit, le musée occupe une position stratégique. Hautement militarisé, le croisement Mathaf-Barbir désigne une zone parmi les plus dangereuses de la ville à cause des francs-tireurs et des enlèvements qui ont lieu autour de la ligne de front. En 1982, se trouvant à la frontière entre Syriens et Israéliens, " le musée lui-même est utilisé comme casernement par les miliciens, et sa façade à la fin du conflit est criblée d'impacts de balles et de roquettes ${ }^{11}$ ». Voici la description des lieux formulée par le responsable de I'UNESCO, Mounir Bouchenaki, lors de sa mission en 1991: "L'intérieur du musée, et notamment les vitrines d'objets de petites dimensions, avait été complètement détruit, et les objets emportés et volés. Le premier directeur du patrimoine libanais après l'indépendance du Liban était l'Émir Maurice Shehab. Il avait eu l'idée géniale de mettre les plus gros objets, par exemple le sarcophage d'Ahiram de Byblos, dans des caissons de béton. Ainsi les œuvres d'art de grande dimension et les mosaïques ont été recouvertes afin d'être protégées. De cette façon, pendant le conflit, même si le musée a été

8 On se réfère aux Accords de Taëf, signés le 22 octobre 1989, et qui ont mis fin à la guerre civile libanaise. 9 FREDDY Raphaël Freddy, HERBERICH-MARX Geneviève, "Le musée, provocation de la mémoire ", Ethnologie française, Hommage d'Ethnologie française à Georges Henri Rivière, nº 1, 1987, p. 87-94.
10 PHARÈS Joseph, "The National Museum of Lebanon ". Museum, vol. 55, n $219-220$, p. 38-43.

11 RICHARD Thomas, Mythologies politiques et identitaires dans les conflits au Moyen-Orient à l'heure de la mondialisation, thèse de doctorat (Sciences politiques), Université d'Auvergne, 2014, p. 109-110. 
sauvagement attaqué, plus de portes, ni de fenêtres, il a toutefois pu résister $d^{\prime}$ une certaine manière. ${ }^{12}$ "

D’après Thomas Richard, les plans de réouverture sont présentés très tôt, c'est-à-dire dès 1992. En raison de " son statut de garant de I'héritage national, de la culture et du rayonnement libanais ${ }^{13}$ ", donner une nouvelle accessibilité au musée est considéré comme une priorité. Mais c'est seulement le 27 novembre 1997, après 5 ans de travaux, qu'a lieu la réouverture officielle du musée, même si encore partielle, en présence du président Elias Haroui. À cette occasion, le choix du parcours muséologique, explique Suzy Hakimian ${ }^{14}$, doit tenir compte de deux éléments: «1) de la nécessité de relier les pièces chronologiquement de manière à constituer un parcours homogène, 2) d'expliquer au public la nature des travaux déjà réalisés et encore en cours ${ }^{15}$ ". Ceci conduit à la création de deux itinéraires: I'un dédié à la « renaissance du musée " avec des œuvres déjà restaurées et mises en lumière, et l'autre " au musée en attente » avec des pièces encore à restaurer qui demeurent dans l'ombre. Ce choix muséographique significatif rend compte des moments sombres vécus par le musée et de la volonté de les ramener progressivement à la lumière. En 2014, après des longues années de travaux, le Musée national a finalement rendu accessible son sous-sol avec des pièces archéologiques importantes et récemment restaurées, encore une fois sans que les traces du conflit soient effacées. La pièce de la mosaïque du "Bon Pasteur ", par exemple, affiche clairement la brèche ouverte par le tir d'un franc-tireur de l'intérieur du musée; une marque dont la reproduction se trouve aussi dans l'installation de l'artiste libanaise Lamia Joreige dédiée au Musée national ${ }^{16}$. En plus de ces signes, d'autres éléments confirment la volonté d'inclure la mémoire du conflit dans le parcours muséal et de la présenter aux visiteurs. On se réfère notamment aux vitrines qui, à la fin du parcours, exposent certaines pièces endommagées pendant le conflit, mais

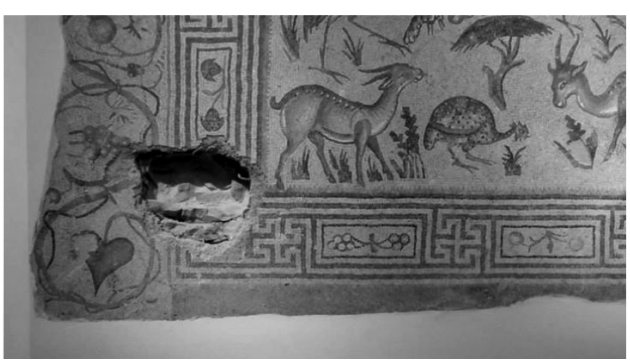

Découverte à Jnah, la mosaique du Bon Pasteur (V ou VI siècle après J.C.) fait partie de la collection du Musée national de Beyrouth. Lors des travaux de restauration, les archéologues ont décidé de garder le trou du mortier qui remonte à l'époque du conflit. aussi à la vidéo documentaire qui, dans une salle à part, retrace l'histoire du musée à l'époque de la guerre et les efforts menés par les employés pour protéger les collections. Aujourd'hui, le Musée national ne cesse de grandir. Un projet d'extension, bénéficiant d'un financement privé, est à l'étude afin de créer de nouveaux espaces ${ }^{17}$. De cette « renais-

12 BOUCHNAKI Mounir, "Patrimoine culturel et conflits récents ", intervention effectuée à l'occasion de la journée d'étude Le patrimoine dans la guerre, organisée par l'association " Mémoires du patrimoine " à l'École du Louvre, le 26 mars 2013.

13 Thomas Richard, op.cit., p. 110.

14 Suzy HAKIMIAN, à l'époque chef de la Section des musées, Musée national de Beyrouth.

15 HAKIMIAN Suzy, National Museum News, $\mathrm{n}^{\circ}$ 6, hiver 1997.
16 On se réfère à l'installation de Lamia Joreige UnderWriting Beirut, Mathaf, 2013.

17 À ce propos, voir: http://www.lorientlejour.com/ article/891182/lancement-du-projet-dextension-du-museenational.html, ainsi que l'article de Robin WRIGHT, "Beirut's Museums of War and Memories ", The New Yorker, 12 octobre 2016: http://www.newyorker.com/news/ news-desk/beiruts-museums-to-war-and-memories. 


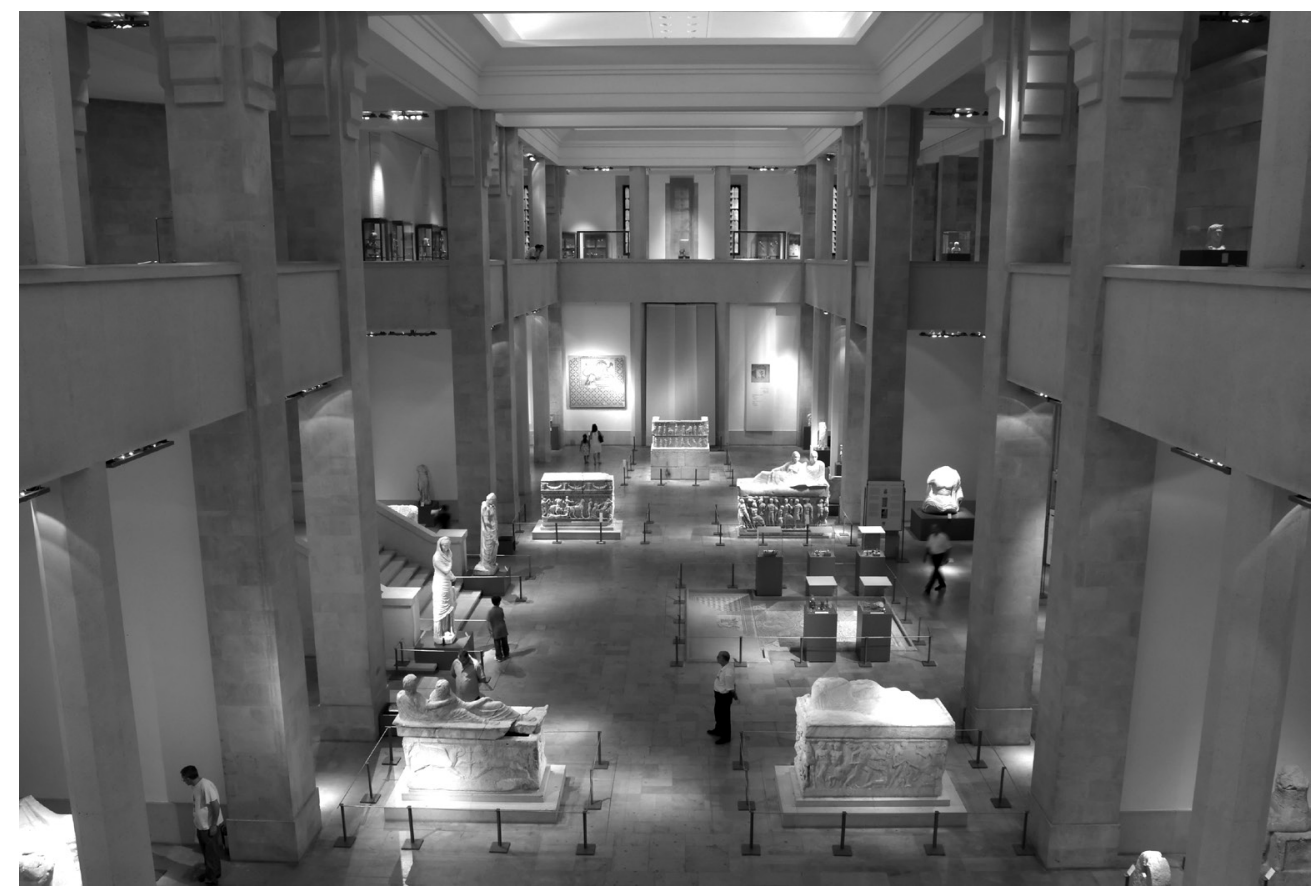

Vue de l'intérieur du musée national de Beyrouth 
sance ", terme souvent associé aussi à Beyrouth, il faut surtout souligner qu'elle a été pensée non pas selon l'ordre de l'avant-guerre, à l'instar du centre-ville, mais plutôt selon celui de l'après. Malgré les difficultés, le musée a composé avec cette phase difficile de son histoire comme avec une pièce encore inconnue, en faisant en sorte que son exposition puisse contribuer à sa compréhension. Un choix courageux, qui a eu pour effet non pas de diminuer, mais de réaffirmer la vocation patrimoniale et nationale du musée qui peut ainsi se projeter vers l'avenir.

\section{De l'immeuble Barakat à Beit Beyrouth}

En remontant la route de Damas, allant du Musée national vers le centre-ville, un autre immeuble mérite notre attention. C'est sans doute son architecture qu'on note avant tout, qui fait de lui une construction remarquable et originale. Cependant, tout regard porté sur la façade de ce bâtiment, connu comme " immeuble Barakat " ou " Maison Jaune ${ }^{18}$ ", ne manquera pas de mesurer aussi les signes importants que le conflit y a imprimés. D'ailleurs, loin d'être banale, cette association entre architecture et conflit est centrale pour comprendre l'histoire, mais aussi le présent de ce lieu. Comme il a été clairement illustré par l'étude de Gregory Buchakjian ${ }^{19}$, outre l'emplacement, la spécificité architecturale de cette construction est précisément ce qui a offert aux francs-tireurs un lieu stratégique d'où contrôler une vaste portion de la ligne de démarcation, tout en restant à l'abri de tirs. Aujourd'hui, cette même combinaison a permis à l'immeuble Barakat d'être préservé de la démolition et restauré pour devenir Beit Beyrouth, le nouveau musée dédié à l'histoire de la ville ${ }^{20}$.

Conçue par l'architecte Youssef Aftimus à la demande de Nicolas et Victoria Barakat ${ }^{21}$ (1924), cette construction a, dès le départ, été pensée comme une structure composée de deux parties identiques donnant I'une dans la rue de Damas, et l'autre dans la rue de l'Indépendance. Initialement composé d'un rez-de-chaussée et d'un premier étage, l'immeuble a subi plusieurs modifications à l'initiative de l'architecte Fouad Kozah. Après la réalisation d'un escalier interne, Kozah prend en charge la construction du deuxième et du troisième étage, ainsi que l'ajout de vérandas (1932). Ces dernières, note Buchakjian, outre qu'elles sont constitutives de la singularité du bâtiment et qu'elles agrandissent l'espace habité, accentuent son lien avec la ville sur laquelle le bâtiment semble s'ouvrir ${ }^{22}$. Mais ce n'est pas tout. «Ce faux péristyle - explique-

18 Cette appellation dérive du nom de la pierre utilisée pour le bâtiment, « la pierre "ramleh" de l'arabe raml ou sable. Cette pierre homogène, légère, solide et isolante est formée de grains de sable maritime, soudés les uns les autres en agglomérats ", KOZAH Fouad, Chroniques d'un architecte du Levant, Paris, L'Harmattan, 2010, p. 115.

19 BUCHAKJIAN Gregory, Habitats abandonnés de Beyrouth, guerres et mutations de l'espace urbain 1860-2015, thèse de doctorat (Histoire de l'art), Université ParisSorbonne - INHA, 2016.
20 À ce propos, voir « Ex nid de snipers, futur lieu de mémoire: visite guidée de Beit Beyrouth ": http://www. lorientlejour.com/article/980518/ex-nid-de-snipers-futurlieu-de-memoire-visite-guidee-de-beit-beirut-video.html 21 Originaires de Syrie, Nicolas et Victoria Barakat s'installent à Beyrouth à la fin du $19^{\mathrm{e}}$ siècle. Ils faisaient partie de la haute bourgeoisie beyrouthine et ils étaient propriétaires d'un magasin de tissus.

22 BUCHAKJIAN Gregory, op. cit., p. 293. 
t-il - constitue un écran, dissimulant l'intérieur de l'immeuble, à la grande satisfaction des combattants qui se l'approprient ${ }^{23}$ ". Comme toute forme d'appropriation, cela ne va pas sans créer des modifications importantes à l'intérieur du bâtiment dans lequel, au premier étage, ont été trouvées des interventions majeures faites par les militaires, comme des barricades et un bunker, conçues afin d'exploiter « au mieux » la structure du bâtiment.

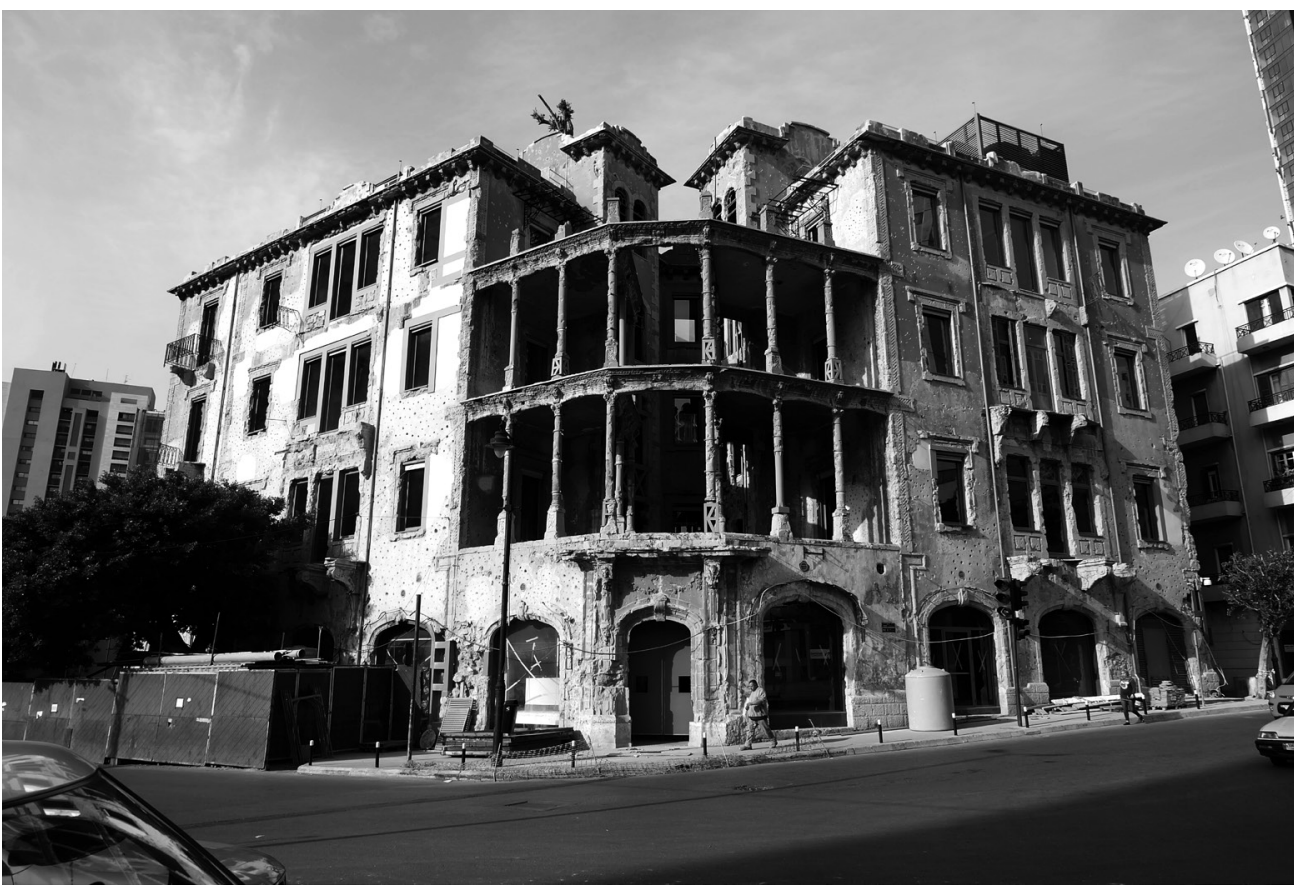

Beit Beyrouth : vue extérieure (c) Youssef Haidar

En 1997, après la fin du conflit, c'est surtout grâce à la persévérance de Mona Hallak ${ }^{24}$, architecte engagée dans la sauvegarde du patrimoine, que le destin de l'immeuble, déjà condamné à la démolition, a été sauvegardé. À la suite de plusieurs années de mobilisation, pendant lesquelles l'immeuble Barakat a fait l'objet de travaux artistiques et de projets culturels ${ }^{25}$, la municipalité de Beyrouth a décidé, en 2003, qu'il serait converti en musée. En 2010, après un accord de coopération avec la ville de Paris, le projet Beit Beyrouth prend forme sous I'aval de

23 Ibid.p. 293.

24 ZADA John, "Hallak on Beit Beirut ", The Planisphere, 8 décembre 2012: http://www.theplanisphere.com/2012/ mona-hallak-barakat-beit-beirut/, Consulté le 10 août 2016.
25 Il faut signaler notamment les projets et les ateliers de recherche menés par les professeurs avec les étudiants de l'ALBA (Académie libanaise des Beaux-Arts), dont celui lancé en 1999 par Pierre Hage-Boutros, Rana Haddad et Gregory Bouchakjian intitulé Bien-fond 1237 ; l'architecte, le vernaculaire et le franc-tireur qui, l'année suivante, a donné lieu à une installation Machines Célibataires en référence à Marcel Duchamp. À ce propos, voir BUCHAKJIAN Gregory, op. cit., p. 300-301. 

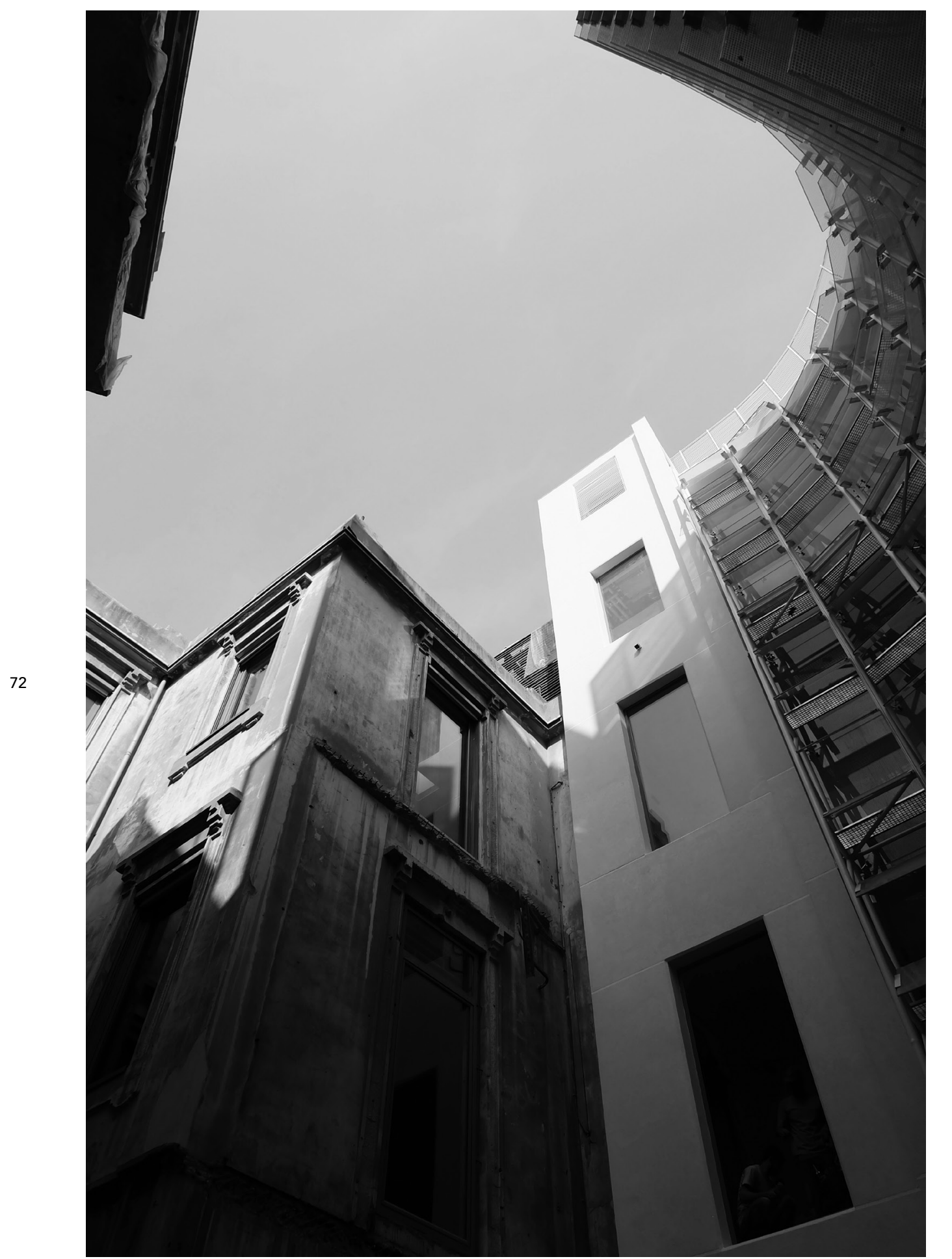

Beit Beyrouth: le bâtiment ancien et le moderne (C) Youssef Haidar 


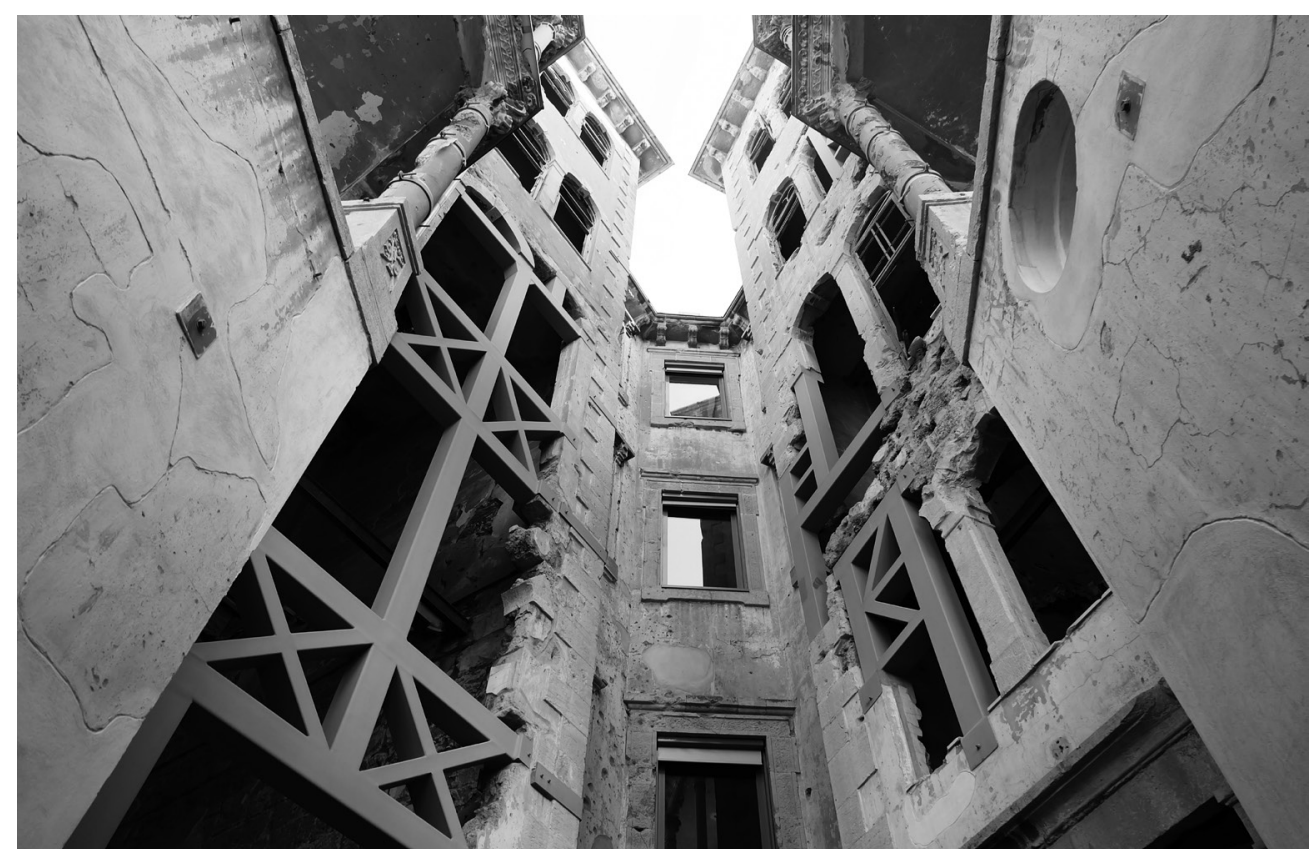

Beit Beyrouth : l'entrée () Youssef Haidar

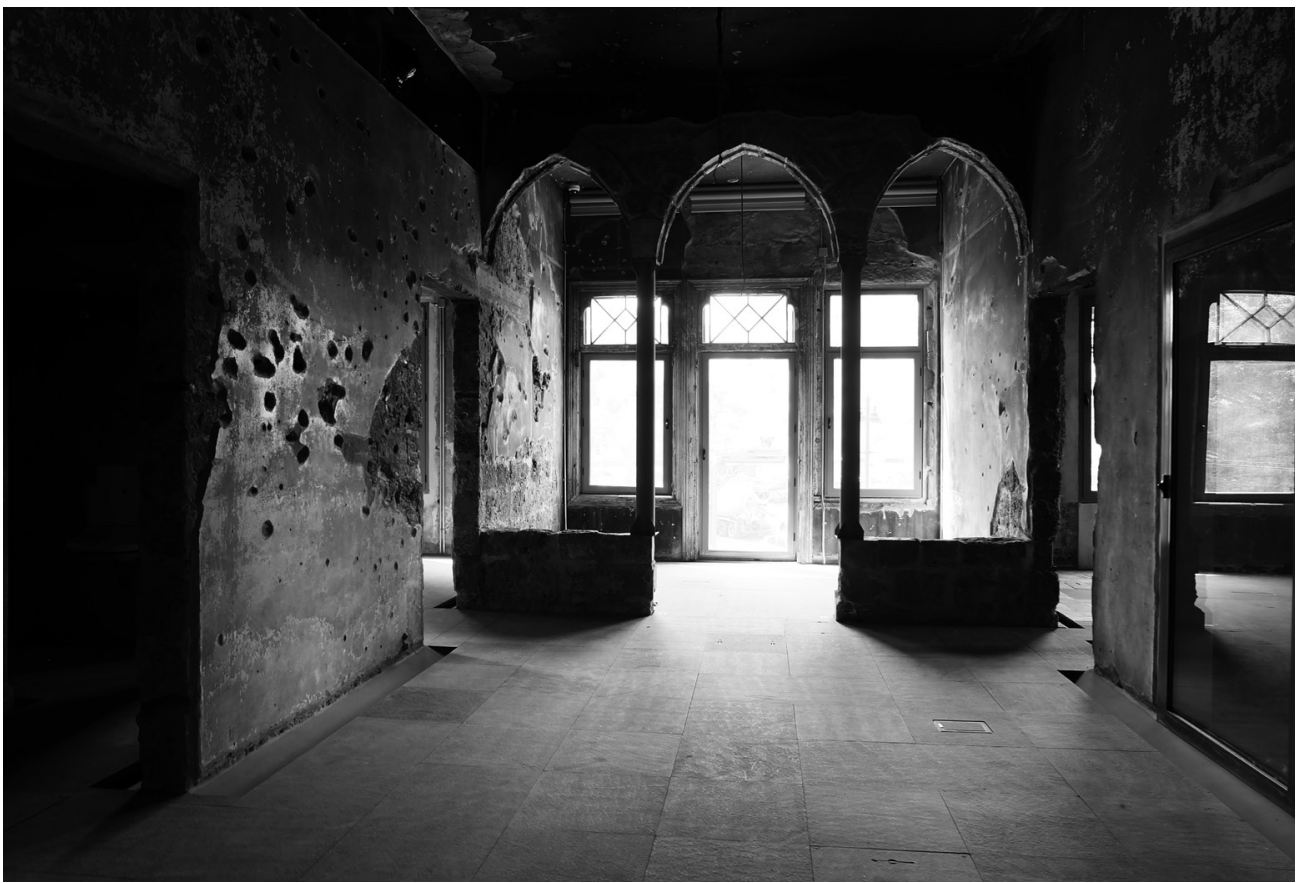

Beit Beyrouth: le $1^{\text {er }}$ étage $($ Youssef Haidar 
Saad Hariri, premier ministre du Liban, et de Bertrand Delanoë, maire de Paris. Depuis, de grands travaux de rénovation ont été menés, non sans retards et quelques critiques, par l'architecte Youssef Haïdar, déjà connu pour son engagement dans les questions patrimoniales concernant la ville.

Aujourd'hui, Beit Beyrouth se présente comme un bâtiment double où la structure originaire est associée à une partie plus moderne. Le travail mené sur la partie historique, explique Haidar, a été pensé de façon à " momifier les lieux » et à " figer l'instant » c'est-à-dire à laisser le tout le plus possible inaltéré et intervenant seulement pour introduire des " prothèses ${ }^{26}$ " là où la structure du bâtiment le demandait. Cette ligne de conduite est d'ailleurs déjà visible depuis l'extérieur du bâtiment dont la surface criblée de balles a été laissée presque intacte, mis à part les supports introduits autour des fenêtres. Il en va de même pour l'accès au bâtiment, resté celui d'origine, où le visiteur peut mesurer les destructions des combattants à partir des débris laissés par la démolition de la cage d'escalier.

Au premier étage, tout a été conservé: les barricades avec les sacs de sable, le bunker, les impacts des balles sur les murs, les ouvertures réalisées pour ouvrir des passages entre les pièces et faciliter la trajectoire des tirs, les graffitis, le carrelage, jusqu'à un vieux lavabo brisé. Considéré comme un mémorial de la guerre à part entière, ce lieu ne contiendra rien d'autre, sinon des panneaux explicatifs permettant aux visiteurs de s'orienter dans l'espace ${ }^{27}$. En revanche, plus significatives ont été les rénovations du deuxième étage. Ici, les graffitis ont été protégés par une plaque transparente, et les signes sur les murs sont encore présents. Les travaux ont rendu l'utilisation des espaces déjà possible. Cet étage, précise Hallak, sera dédié à l'histoire du développement urbain de la ville et de la société beyrouthine de l'époque ottomane jusqu'à aujourd'hui ${ }^{28}$. Le troisième et dernier étage, entièrement rénové aussi en raison de son état avancé de dégradation, a été reconfiguré comme une salle d'exposition de $800 \mathrm{~m}^{2}$, et il sera destiné à accueillir des expositions d'art (photographie, peinture et sculpture) portant sur la ville de Beyrouth. Ce parcours, explique Haidar, voudrait aussi symboliser une sorte de "libération " progressive, ou de diminuendo vis-à-vis de la référence au conflit: du premier étage, où l'état de conservation des lieux dégage aussi une forte charge émotionnelle, au dernier étage qui se présente comme un lieu épuré. Quant à la partie moderne, incluant également un sous-sol avant inexistant, elle a été conçue afin d'accueillir le personnel du musée et les chercheurs. On y trouve les services, un centre de documentation et un auditorium. Ce qui répond à la deuxième vocation de ce lieu, celle de centre culturel.

26 Ce sont les termes employés par Youssef Haidar lors de la visite guidée effectuée pour le quotidien libanais L'Orient, le jour : http://www.lorientlejour.com/article/980518/ex-nid-desnipers-futur-lieu-de-memoire-visite-guidee-de-beit-beirutvideo.html.
27 Lors de ma visite de Beit Beirut (juillet 2016), les panneaux n'étaient pas encore affichés. 28 ZADA John, "Hallak on Beit Beirut ", op.cit. 


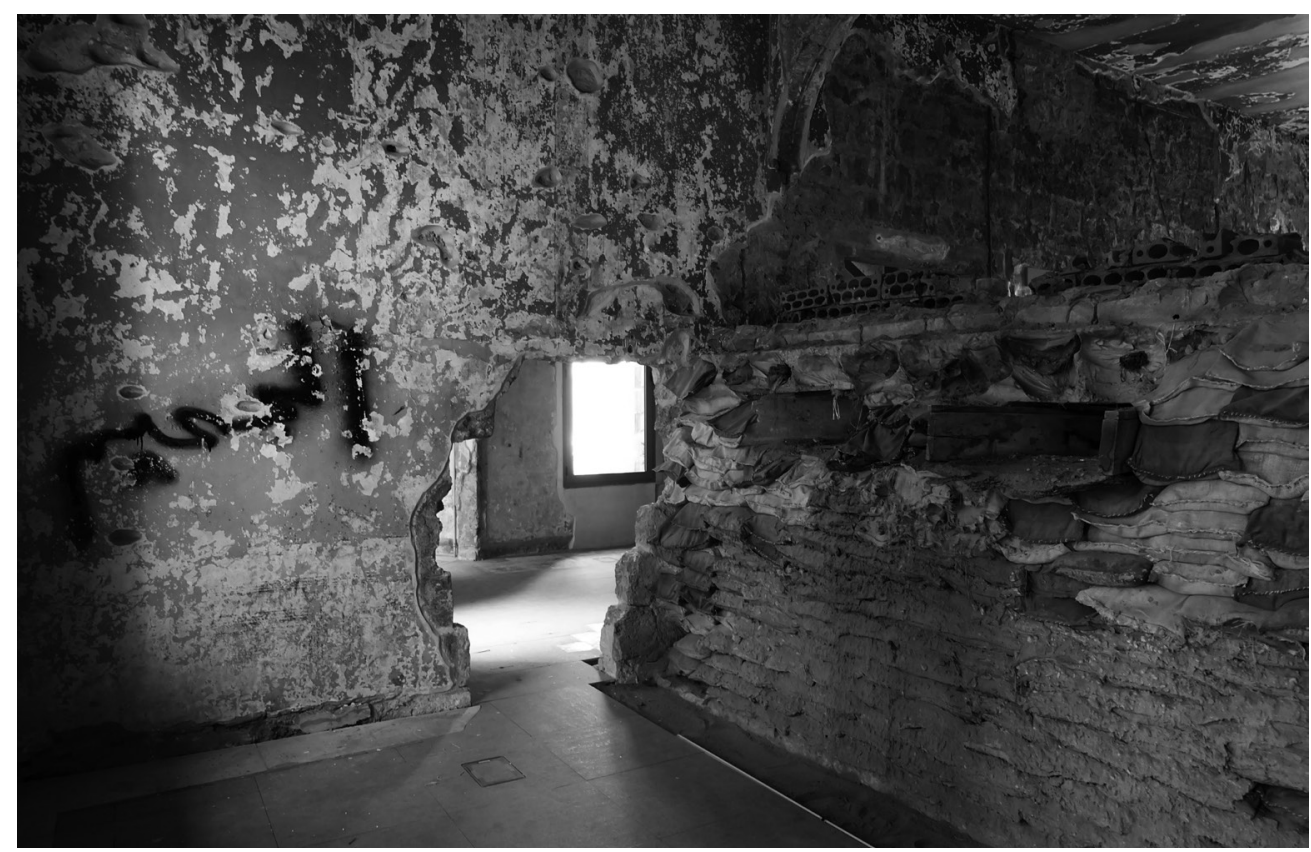

Beit Beyrouth: le bunker au $1^{\text {er }}$ étage $\odot$ Youssef Haidar

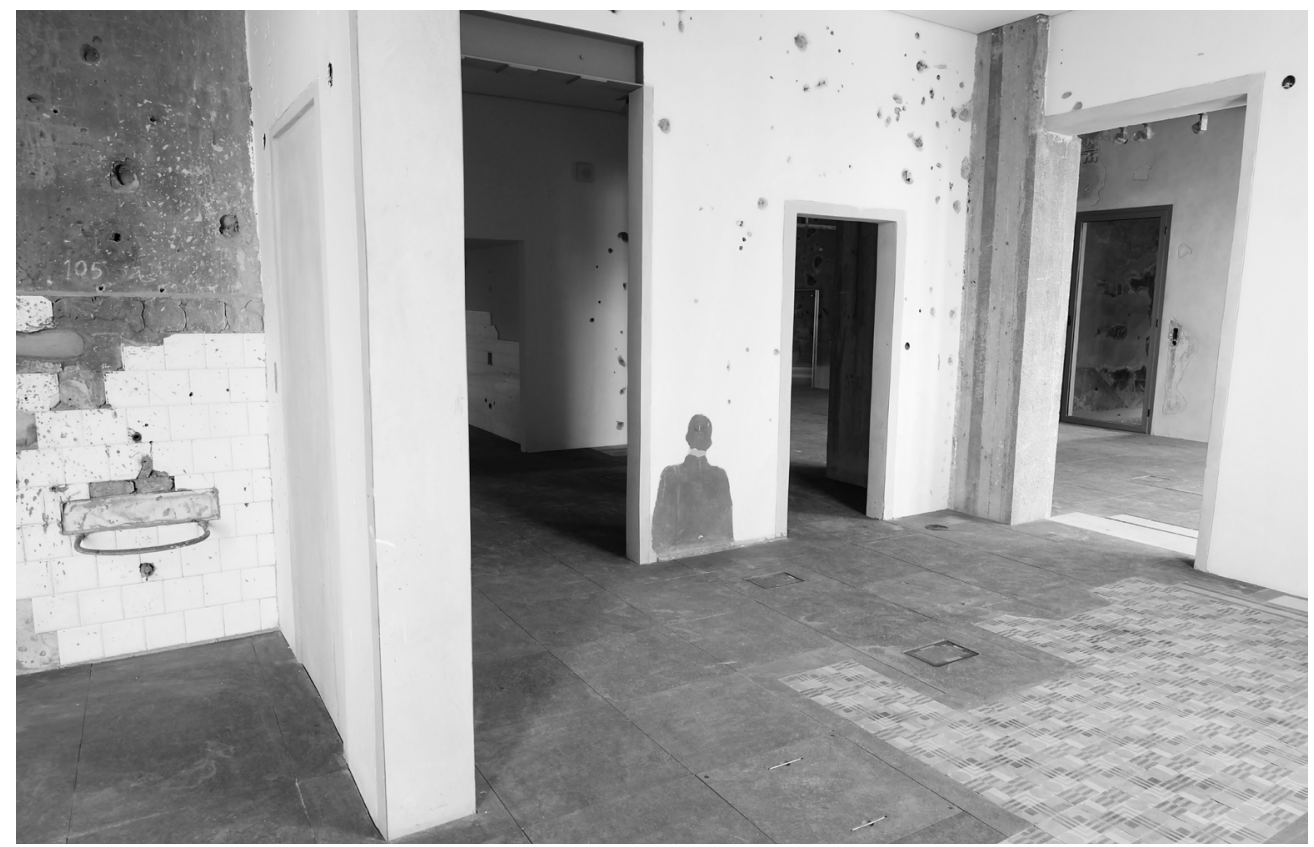

Beit Beyrouth: le $2^{\text {ème }}$ étage (C) Youssef Haidar 


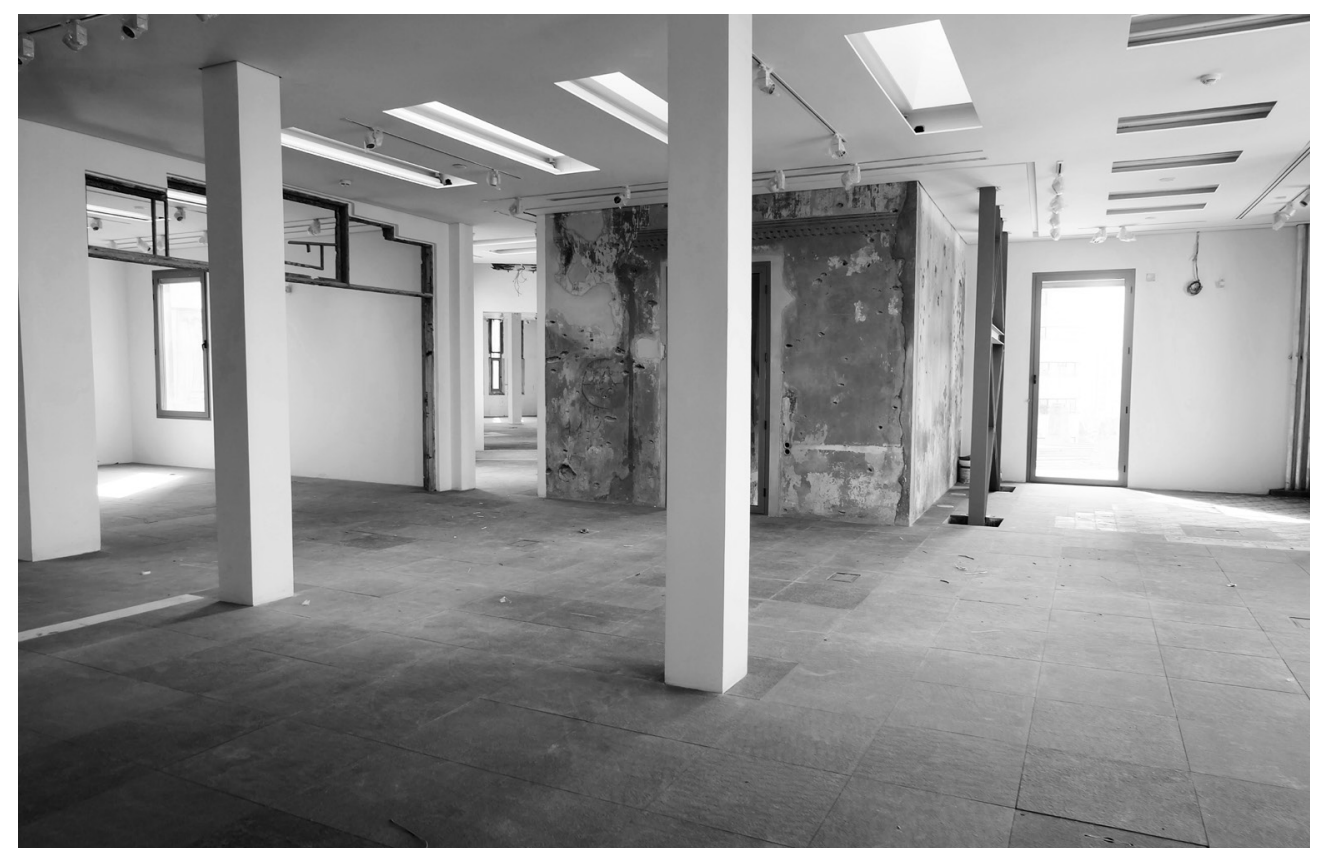

Beit Beyrouth: le $3^{\text {ìme }}$ étage (C) Youssef Haidar 
La conversion de ce bâtiment en musée constitue donc un exemple vertueux de sauvegarde du patrimoine. Elle nous permet surtout de saisir le croisement entre les enjeux mémoriels et patrimoniaux actuels à Beyrouth. Cela dit, cette transformation ne va pas sans poser des questions, notamment à propos des différents usages dont Beit Beyrouth fera l'objet. En effet, malgré le travail important de rénovation mené au deuxième et au troisième étage, afin de leur attribuer une nouvelle fonction, il reste que toute exposition sera toujours en relation avec la force mémorielle dégagée par ces salles, notamment par le lien qu'un parcours muséographique installe entre les pièces et l'espace qui les accueille. Le risque est en effet celui de la normalisation ou de la banalisation d'un lieu où, jusqu'à présent, tout a été mis en œuvre afin d'en préserver le plus possible le caractère originaire. Autrement dit, si dans le cas du Musée national le défi relevait de l'incorporation de la mémoire du conflit dans l'institution muséale, pour Beit Beyrouth, la situation s'avère renversée, l'enjeu étant de créer un espace d'exposition institutionnel à l'intérieur d'un lieu de mémoire. Dans les deux cas, l'intention est clairement pédagogique et la provocation de la mémoire dont ces lieux se font porteurs " a comme objectif d'éveiller au civisme les jeunes générations dans le dessein d'éviter le retour à la barbarie ${ }^{29}$ ". Cela dit, et comme l'explique Dominique Poulot, il semble difficile de voir dans cette volonté de transmission, signifiée notamment par l'ouverture d'un musée ${ }^{30}$, « la conclusion apparemment heureuse de maintes "guerres de mémoires" contemporaines ${ }^{31}$ ". Le souhait est, au contraire, que ces musées, rendant ces deuils et ces souvenirs de guerre des "souvenirs nationaux", comme le dirait Renan, puissent impulser un processus de réélaboration profonde, collective et historique, de ces événements, et donner lieu à " l'effort du commun ${ }^{32}$ ».

29 BOURSIER Jean-Yves, « D'une guerre à l'autre en Europe ", Musées de guerre et mémoriaux, (BOURSIER Jean-Yves dir.), Paris : Éditions de la maison des sciences de l'homme, 2006, p. 10.

30 À ce propos, il est important de préciser que le musée Beit Beirut n'a toujours pas été inauguré officiellement. Lors de ma visite (juillet 2016), l'ouverture officielle était prévue sous peu (septembre 2016). À ce jour (11 avril 2017), nous demeurons toujours en attente. Il serait sans doute intéressant de suivre l'évolution de cette situation, métaphore involontaire de l'état de suspension mémorielle qui caractérise le pays depuis plusieurs années.
31 POULOT Dominique, « Musée et guerres de mémoires: pédagogie et frustration mémorielle ", (BLANCHARD Pascal dir.). Les guerres de mémoires, « Cahiers libres ", Paris: La Découverte, 2008, p. 230.

32 FOREST Philip, RENAN Ernest, Qu'est-ce qu'une nation? Paris: P. Bordas, 1991. 